\title{
DETECTION OF ECOLOGICAL DISTURBANCES TO SEABED FAUNA THROUGH CHANGE OF WEIGHT DISTRIBUTION
}

\author{
Mayumi Naka*, Ritei Shibata* and Ross Darnell**
}

\begin{abstract}
The effect of trawling on seabed fauna in the Northern Prawn Fishery experimental region of Australia is investigated through distributional changes in individual weights for each species. A stochastic growth model is employed to overcome a limited number of effective observations. One statistical challenge is to deal with non-identically distributed observations as only total weights and numbers of individuals caught for each species are observed. A modified Cramér-von Mises statistic is introduced and the $p$-values are evaluated by random number generation. As a result, the gamma distribution, the equilibrium distribution of the stochastic growth model fits well to 57 out of 80 cases before trawling. We conclude that most of the species are unaffected by trawling but several other species are shifted towards lighter weights. The unevenness of the effect over regions suggests that other environmental effects and ecological factors are involved.
\end{abstract}

Key words and phrases: Cramér-von Mises statistic, gamma distribution, stochastic growth model.

\section{Introduction}

Effects of various methods of harvesting the sea have been investigated in many articles on marine ecology. Collie et al. (2000) carried out a meta-analysis of 39 published fishing impact studies to draw general conclusions. Bishop et al. (2000) investigated the impact of technology on vessel performance in a trawl fishery during 1988-96 by using a generalized estimating equation. Burridge et al. (2003) investigated trawl-depletion rate for benthic fauna in an area closed to commercial trawling. In this paper we detect the effect of trawling through changes of weight distribution, the equilibrium distribution of a stochastic growth model. The stochastic growth model is frequently used for modelling population size (Russo et al. (2009)) or size of plants (Rupsys and Petrauskas (2007)) or animals (Tovar-Ávila et al. (2009)). It is shown that the equilibrium distribution of the model fits well as a reference distribution when no effective ecological disturbance exists. This result allows us to detect any effective disturbance by departure from the gamma distribution identified before the disturbance. The reason why we focus on individual weight as an index of disturbance in this paper is that it is sensitive to any ecological disturbances and easy to measure compared to size. An advantage of our approach is to be able to draw a whole

\footnotetext{
Received February 23, 2012. Revised June 27, 2012. Accepted October 15, 2012.

*Keio University, 3-14-1 Hiyoshi, Kohoku-ku, Yokohama 223-8522, Japan.

**CSIRO Mathematics, Informatics and Statistics, EcoSciences Precinct, 41 Boggo Road, Dutton Park, QLD 4102, Australia.
} 
picture of the current status of each species on seabed before and after trawling without introducing any particular estimating equation or indices. The data used in this paper is that obtained in the project "Quantifying the effects of trawling on seabed fauna in the Northern Prawn Fishery" in Australia, which will be explained in detail in Section 2. Exhaustive analyses of the data were already reported in Haywood et al. (2005). After calculating various fundamental statistics and drawing many graphs and maps, they tried to find out the effect of trawling by application of a simple depletion and recovery model. Unfortunately it does not seem successful. There are several reasons why their analysis was not successful. One is that it is a class by class analysis, using popular descriptive statistics and plots. Our result shows that class by class analysis is too coarse to detect any effect of ecological disturbances. Instead we detect such disturbances by building up species by species stochastic growth models. Another reason is that their analysis is based on the whole weight of each species caught, normalised by the dredge area. The biomass density is a useful abundance measure for each survey area from the view point of fishery but not so for the detection of ecological disturbances. Changes of individual weight would be more useful for detecting ecological disturbance. Then, we detect it in this paper by fitting a stochastic growth model for individual weight. A technical problem which arises in analysing unnormalised weight with the number of catches of each species is that we have to deal with non identically distributed variables. This does not cause any serious problem in parameter estimation but requires a modification of the goodness of fit test statistic, most of which are proposed for i.i.d. (independent and identically distributed) observations. We evaluate $p$-values of a modified Cramér-von Mises statistic by executing computer simulations around the maximum likelihood estimate since even the asymptotic distribution is unknown. The stochastic growth model is introduced in Section 3 and a gamma distribution is derived as a stationary distribution for the weight. Distribution before trawling is identified in Section 4. The effect of trawling is detected through change of distribution in Section 5. Comparison with Welch's $T$ test is given in Section 6 .

\section{Seabed fauna in Northern Prawn Fishery and data}

The data is from the Fisheries Research and Development Corporation (FRDC) funded Project 2002/102, "Quantifying the effects of trawling on seabed fauna in the Northern Prawn Fishery" (NPF) in Australia. The project was originally identified as a high priority research area by the Northern Prawn Fishery Management Advisory Committee (NORMAC) because under the Environmental Protection and Biodiversity Conservation Act (EPBC Act), Australian fisheries are required to demonstrate their environmental sustainability. Industry offered special funding to support the research and the FRDC was asked to manage the project. The Commonwealth Scientific and Industrial Research Organisation (CSIRO) agreed to carry out the work, develop the scope of the work and the experimental design and contribute to the funding.

Trawlers in the NPF tend to concentrate their fishing on areas of higher 
Table 1. Part of the data.

\begin{tabular}{cccclrc}
\hline Region & Plot & Treatment & Time & \multicolumn{1}{c}{ Scientific name } & Count & Weight $(\mathrm{g})$ \\
\hline East & 12 & 4 & Before & Retiflustra cornea & 1 & 0.25 \\
East & 12 & 4 & Before & Melaxinaea vitrea & 1 & 9.16 \\
East & 12 & 4 & Before & Tubeworm OPNO 006 & 14 & 2.28 \\
East & 12 & 4 & Before & Neritidae OPNO 142 & 0 & 0 \\
East & 12 & 4 & Before & Leucosia whitei & 1 & 1.49 \\
\hline
\end{tabular}

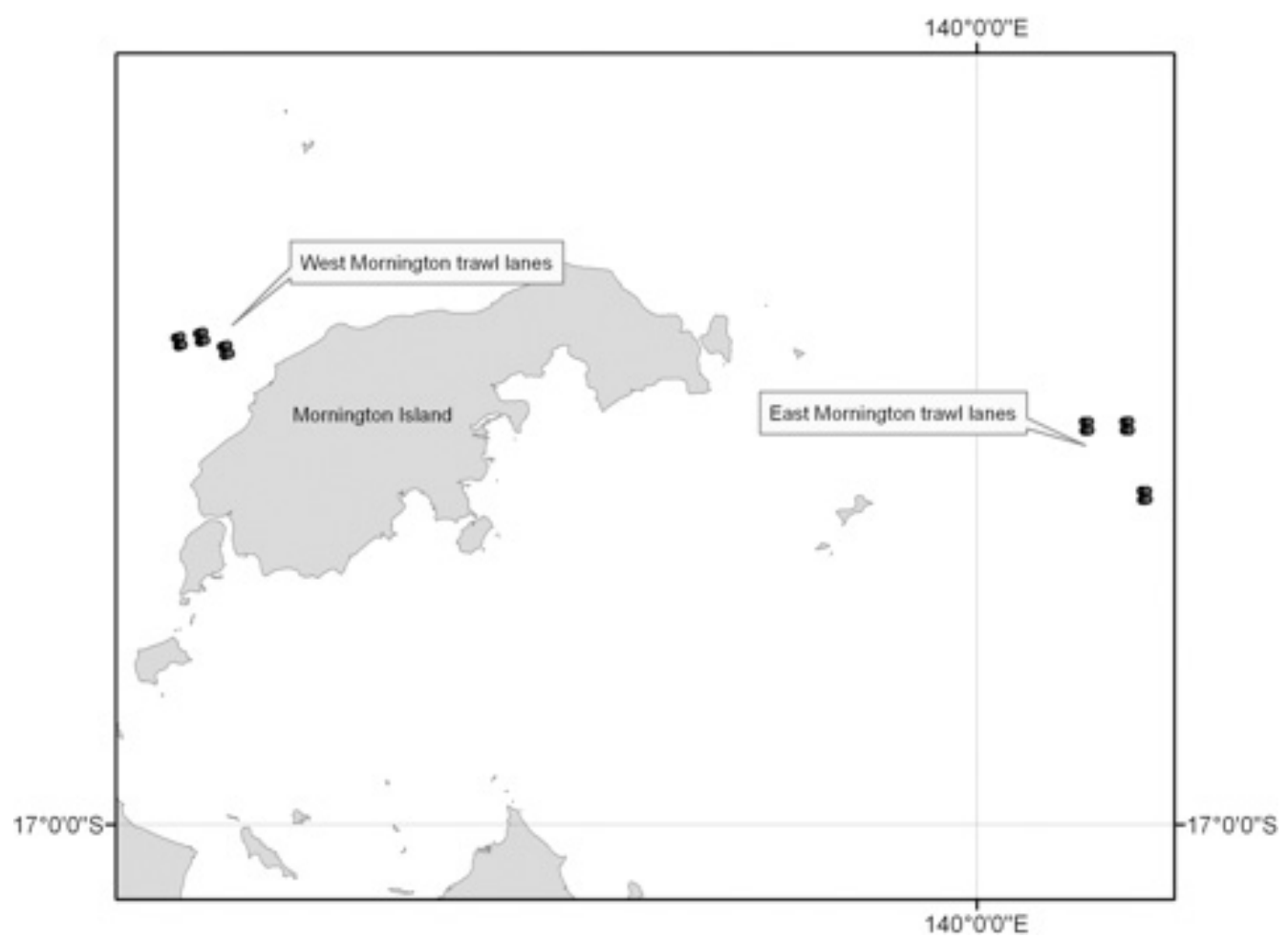

Figure 1. Two regions near Mornington Island used for the experimental survey (Haywood et al. (2005)).

prawn density. Also intensive trawling of small areas is a feature of the tiger and endeavour prawn fishery. Around $20 \%$ of the catch is prawns but the rest are animals collected from the seabed. Such by-catch animals can be seen in Table 7 , although those are not caught by trawling but by experimental dredges.

Table 1 shows part of the data, which consists of 207,726 records, obtained by the experimental dredge survey for an explanation of the data structure. The first column labelled Region, indicates the region where the experiment was performed. The survey area shown in Fig. 1 is roughly divided into two regions, East (East of Mornington Island) and West (West of Mornington Island); three plots are set in each region, Plots 3, 5 and 6 in the West and Plots 9, 10, and 12 in 
the East. Geographical features of the seabed of East and West are different; East is deeper but West is rougher and harder acoustically (Haywood et al. (2005)). Such a difference suggests the need of separate analyses for the East and West regions. At each plot, three levels of experimental trawlings (Treatments) were repeated three times. The three levels are the intensity of trawling, 0,4 , and 20 , and the number of repeated trawlings on each plot. The trawled sea bed were dredged immediately after, or 6,12 or 18 months after trawling as well as before trawling, indicated by the variable Time. Scientific Name is the name of the species caught by each dredge and Count is the number of individuals of each species caught in each trawl. Weight is the total weight of each case in grams.

Although the primary aim of the survey was to investigate the effect of different levels of trawl intensity and recovery time, we will concentrate on whether the effect of trawling is significant, since the number of effective observations is not large enough for a detailed analysis because of the large number of empty catches. Therefore, in this paper the treatment levels 4 or 20 are combined, and the weights recorded immediately after trawling are used for analysis in contrast with the weights before trawling. Although some useful information might be lost by combining those levels, it is a trade-off between the number of effective observations and the sharpness of the result. We may satisfy ourselves if the effect of trawling were detected in a systematic manner. Although 778 species were observed in this survey, only 16 classes of species were considered for analysis as there were too many zero catches for the other classes. Furthermore, 5 of the 16 classes are not appropriate for analysis. Demospongiae is hard to count since they are colonial, Pisces can easily escape from dredging and trawling, and Phaeophyta, Liliopsida and Chlorophyta are fragile plants difficult to collect intact. Eleven classes remained for our investigation: Hydorozoa, Anthozoa, Gymnolaemata, Polychaeta, Bivalvia, Gastropoda, Asteroidea, Ophiuroidea, Echinoidea, Crustacea and Ascidiacea. As a result 76 species remained in those classes for analysis, but we also removed those species with observations of less than 5, while the maximum number of observations for each case is 27 before trawling and 18 after trawling. We will report elsewhere other analyses of the data, for example, the number of individuals caught.

\section{Weight distribution}

\subsection{Stochastic growth model and the equilibrium distribution}

Richards (1969) shows that many of the deterministic growth models modify the relative growth rate $(1 / X) d X / d t$,

$$
\frac{d X_{t}}{X_{t}}=f\left(X_{t}\right) d t
$$

where $f\left(X_{t}\right)$ is a function of the animal weight $X_{t}$ at time $t$. An example of a deterministic model is the logistic,

$$
d X_{t}=\rho X_{t}\left(\kappa-X_{t}\right) d t
$$


where $\kappa$ is the growth limit and $\rho$ is an exponential rate constant. It is one of the well known models for population growth (Davidson (1938), Smith (1963)), probably first proposed by Verhulst (1838). This model has since been used for describing many other aspects of growth other than that related to the population, for example, Marubini et al. (1972) analysed the growth of boys' and girls' heights.

We introduce a stochastic growth model for the individual's weight for each seabed fauna species at time $t$,

$$
d X_{t}=r X_{t}\left(1-\frac{X_{t}}{k}\right) d t+\sigma X_{t} d B_{t}
$$

where $r$ is the growth rate, $k$ is the growth limit and $B_{t}$ is a standard Brownian motion. Model (3.2) is a stochastic version of (3.1) but unlike (3.1) does not assume growth is monotonically increasing with time. In this respect the stochastic growth model (3.2) is a better model for weight. To explain the relation between these two models, the derivation by May (1973) is appealing. He assumed that the growth limit $\kappa$ randomly fluctuates as $\kappa=k+\gamma(t)$ reflecting environmental changes for plants and animals. If it is written as

$$
(\kappa-k) d t=\sigma_{0} d B_{t},
$$

then (3.1) becomes

$$
d X_{t}=(\rho k) X_{t}\left(1-\frac{X_{t}}{k}\right) d t+\left(\rho \sigma_{0}\right) X_{t} d B_{t},
$$

which is equal to the model (3.2) when $r=\rho \kappa$ and $\sigma=\rho \sigma_{0}$. The source of random fluctuation in this derivation is that of the growth limit $\kappa$. In other words, individual difference comes from different values of $\kappa$ in this model.

In this paper, we are interested in investigating the distributional change of the weight $X_{t}$ rather than tracing the growth of individual weight. Let $p(x, t)$ be the probability density function of $X_{t}$. Then, as is shown in the Appendix, regardless of the shape of $p(x, t)$ it converges to the equilibrium distribution $p(x)$ as time $t$ goes on, provided that $r>\sigma^{2} / 2$ (p. 629, May (1973)). Note that the way of understanding a stochastic differential equation is a bit different from that of a non-stochastic differential equation. The random variable $X_{t}$ describes the weight of individuals in the population at time $t$ as a whole. Thus $p(t, x)$ is the distribution for whole individuals in the population at time $t$. Since the equilibrium distribution $p(x)$ is the distribution at the stage of equilibrium, $p(t, x)$ quickly goes back to $p(x)$ even if $p(x)$ were slightly modified by birth and death in the population. Of course, it takes longer to come back to the equilibrium if any big disturbance from outside occurs. Except for such a disturbed case, the equilibrium distribution can be used as a distribution of the weight, as far as $X_{t}$ follows the stochastic differential equation (3.2). Therefore, it would be reasonable to use this equilibrium distribution $p(x)$ as a model for the weight of each species in a region where no disturbance from outside were made for an 
adequate period as far as the individual weight increases or decreases along with the equation (3.2).

As has been suggested by May (1973), the equilibrium distribution of $X_{t}$ is a gamma distribution $\mathrm{G}_{\mathrm{A}}(\nu, \alpha)$ with the shape parameter $\nu=2 r / \sigma^{2}-1$ and the scale parameter $\alpha=\sigma^{2} k / 2 r$. As a reference, we give a simple proof for the convergence to the equilibrium distribution as time tends to infinity, with necessary conditions in the Appendix. The proof is similar to that of Goel and Richter-Dyn (1974), but the conditions are much simpler. It is worth noting that the solution does not remain the same if the definition of the stochastic integral is not an Ito type integral. The equilibrium distribution is not necessarily gamma if other integral types, such as the Stratanovich integral were employed (Feldman and Roughgarden (1975)).

It is of course possible to introduce another model for the weight. For example, a deterministic model

$$
d X_{t}=\rho \kappa X_{t}^{2 / 3} d t-\rho \kappa^{2 / 3} X_{t} d t
$$

seems reasonable for the weight $X_{t}=Y_{t}^{3}$, where the length $Y_{t}$ follows the von Bertalanffy model,

$$
Y_{t}=Y_{\infty}-\left(Y_{\infty}-Y_{0}\right) \exp (-\beta t)
$$

with $\rho=3 \beta / Y_{\infty}^{2}, \kappa=Y_{\infty}^{3}$. The differential equation (3.3) does a good job describing the difference between surface-proportional anabolism and weightproportional catabolism (von Bertalanffy (1960)). We have tried to fit a stochastic modification of (3.3) to the given data,

$$
d X_{t}=\rho \kappa X_{t}^{2 / 3} d t-\rho \kappa^{2 / 3} X_{t} d t+\sigma X_{t} d B_{t}
$$

However, it did not work well. One of the reasons why it does not work well is the von Bertalanffy model is a deterministic model for the size, so that it is appropriate for the length of plant or any other increasing size but not for the weight which increases or decreases. In fact, the equilibrium distribution of the stochastic model (3.4) is a power transformed gamma distribution with the power $-1 / 3$. Such a negative power transformed gamma distribution does not seem reasonable. Also, the use of such a distribution for which no reproducibility property holds true causes a lot of problems in the estimation of parameters and test of goodness of fit. The distribution of the total weight of each case, which is the only available observation, becomes much more complicated. Although it is a big challenge to develop any other type of stochastic modification of the von Bertalanffy model, we concentrate ourselves on the model (3.2) in this paper.

\subsection{Observed weight and maximum likelihood estimate}

As has been mentioned, only total weights for each species in each dredge were recorded in this survey since individual weight measurement is time consuming and expensive. Let $W_{i}$ represent the sum of the unobserved individual weights, $X_{j}$, so

$$
W_{i}=X_{1}+X_{2}+\cdots+X_{m_{i}}, \quad i=1, \ldots, N
$$


where $m_{i}$ is the number of individuals caught in Case $i$. Note that $X_{j}$ 's are not observable but $W_{i}$ 's are observed with $m_{i}$ 's. The observations $W_{1}, W_{2}, \ldots, W_{N}$ are now thought to be independent but not identically distributed. However, we can avoid other complications because of the reproducibility of the gamma distribution. That is, the $W_{i}$ is still distributed as gamma $\mathrm{G}_{\mathrm{A}}\left(m_{i} \nu, \alpha\right)$, provided that $X_{j}, j=1,2, \ldots, m_{i}$, are independent and identically distributed as $\mathrm{G}_{\mathrm{A}}(\nu, \alpha)$, i.e. all individuals share the same scale $\alpha$, and shape $\nu$ parameters. The maximum likelihood estimate of $\alpha$ is a function of $\nu$,

$$
\hat{\alpha}=\frac{w}{m \nu},
$$

where $w=\sum_{i=1}^{N} w_{i}$ is the sum of observed total weights $w_{1}, w_{2}, \ldots, w_{N}$ and $m=\sum_{i=1}^{N} m_{i}$ is the sum of the number of individuals observed. Although no closed form is known for the maximum likelihood estimate of $\nu$ and $\alpha$, we could obtain the numerical value of the estimate of $\nu$ by a numerical algorithm to maximise the profile likelihood,

$$
L(\nu, \hat{\alpha})=\sum_{i=1}^{N}\left\{-\log \left(\Gamma\left(m_{i} \nu\right)\right)+\left(m_{i} \nu-1\right) \log w_{i}\right\}-m \nu \log \left(\frac{w}{m \nu}\right)-m \nu .
$$

The function $n \operatorname{lminb}$ is an implementation of the nonlinear minimisation program developed at AT\&T Bell Laboratories on $\mathrm{R}$ and available in any version of $\mathrm{R}$ as a part of standard library stats. The version of $\mathrm{R}$ we have used is 2.12.2, which is available from http://cran.r-project.org/.

\subsection{Goodness of fit}

Goodness of fit tests are indispensable when a model is fitted to data. One visual diagnostic tool is the quantile-quantile or Q-Q plot. However it is restricted to the case of independent and identically distributed observations. A similar visual diagnostic tool applicable to non-identically distributed observations would be a probability-probability or P-P plot (Gan and Koehler (1990), Holmgren (1995)) which is called the "Universal Q-Q plot" by Luceño (2007). The P-P plot for $N$ independent observations $x_{1}, x_{2}, \ldots, x_{N}$, each of which is distributed as $F_{k}\left(x_{k} ; \boldsymbol{\theta}\right), k=1,2, \ldots, N$, in our case which is gamma distribution with the parameter $\left(m_{k} \nu, \alpha\right)$ for the common parameter $\boldsymbol{\theta}=(\nu, \alpha)$, is a plot of the $N$ points,

$$
\left(\frac{k-0.5}{N}, F_{(k)}\left(x_{(k)} ; \boldsymbol{\theta}\right)\right), \quad k=1,2, \ldots, N
$$

where $x_{(1)}, x_{(2)}, \ldots, x_{(N)}$ are order statistics of the observations and the distributions $F_{k}\left(x_{k}, \boldsymbol{\theta}\right), k=1,2, \ldots, N$, are arranged in the same order. The estimate $\hat{\boldsymbol{\theta}}$ is plugged into $\boldsymbol{\theta}$ if the parameter is unknown. A numerical goodness of fit statistic parallel to the $\mathrm{P}-\mathrm{P}$ plot is then

$$
\tilde{W}_{N}^{2}(\boldsymbol{\theta})=\frac{1}{12 N}+\sum_{k=1}^{N}\left(F_{(k)}\left(x_{(k)} \boldsymbol{\theta}\right)-\frac{k-0.5}{N}\right)^{2},
$$


where the empirical estimate, $\tilde{W}_{N}^{2}(\hat{\boldsymbol{\theta}})$, is used if the parameters are unknown as the same as in the P-P plot. This statistic is closely related to the Cramér-von Mises statistic,

$$
\begin{aligned}
W_{N}^{2}(\boldsymbol{\theta}) & =N \int_{-\infty}^{\infty}\left(F_{N}(x)-F(x ; \boldsymbol{\theta})\right)^{2} d F(x ; \boldsymbol{\theta}) \\
& =\frac{1}{12 N}+\sum_{k=1}^{N}\left(F\left(x_{(k)} ; \boldsymbol{\theta}\right)-\frac{k-0.5}{N}\right)^{2}
\end{aligned}
$$

where $F_{N}(x)$ is the empirical distribution function and reduces to $\tilde{W}_{N}^{2}(\boldsymbol{\theta})$ when the observations are independent and identically distributed as $F(x ; \boldsymbol{\theta})$. There are many articles on the behaviour of $W_{N}^{2}(\hat{\boldsymbol{\theta}})$ (Sukhatme (1972), Lockhart and Stephens $(1985))$ as well as that of $\tilde{W}_{N}^{2}(\hat{\boldsymbol{\theta}})$ (Pierce and Kopecky (1979), Loynes $(1980))$. However, their results are not directly applicable for obtaining the $p$ value of $\tilde{W}_{N}^{2}(\boldsymbol{\theta})$ or $\tilde{W}_{N}^{2}(\hat{\boldsymbol{\theta}})$ in our case, so that we will evaluate the $p$-value of $\tilde{W}_{N}^{2}(\boldsymbol{\theta})$ or $\tilde{W}_{N}^{2}(\hat{\boldsymbol{\theta}})$ for the given observation based on 500 sets of generated random numbers which are distributed as $\mathrm{G}_{\mathrm{A}}\left(m_{i} \nu, \alpha\right), i=1,2, \ldots, N$. The evaluation is not only at the point estimate $\left(m_{i} \hat{\nu}, \hat{\alpha}\right)$ but also at several lattice points in the neighbourhood, $\left(m_{i} \nu, \alpha\right)$ for $\nu=0.5 \hat{\nu}, 0.75 \hat{\nu}, 1.25 \hat{\nu}$ and $1.5 \hat{\nu}$ and $\alpha=0.5 \hat{\alpha}$, $0.75 \hat{\alpha}, 1.25 \hat{\alpha}$ and $1.5 \hat{\alpha}$. Table 2 shows one of the examples of the $p$-values in the neighbourhood. The data is for the case of Case 2 in the table in the Appendix. As is easily seen, the $p$-value does not fluctuate so much, ranging from 0.091 to 0.133 , so that, we use the minimum in the neighbourhood as a $p$-value through this paper, which is favourable to the rejection of the fit.

Table 2. $p$-values of the goodness of fit statistic for Case 2 .

\begin{tabular}{rccccc}
\hline & $0.5 \hat{\alpha}$ & $0.75 \hat{\alpha}$ & $\hat{\alpha}$ & $1.25 \hat{\alpha}$ & $1.5 \hat{\alpha}$ \\
\hline $0.5 \hat{\nu}$ & 0.119 & 0.106 & 0.108 & 0.121 & 0.096 \\
$0.75 \hat{\nu}$ & 0.125 & 0.127 & 0.126 & 0.114 & 0.114 \\
$\hat{\nu}$ & 0.129 & 0.128 & 0.125 & 0.108 & 0.133 \\
$1.25 \hat{\nu}$ & 0.115 & 0.106 & 0.114 & 0.118 & 0.122 \\
$1.5 \hat{\nu}$ & 0.115 & 0.091 & 0.099 & 0.103 & 0.092 \\
\hline
\end{tabular}

\section{Distribution before trawling}

A gamma distribution is fitted to each observation before trawling. The results for all species are shown in Table 7 in the Appendix, where the gamma distribution $\mathrm{G}_{\mathrm{A}}(\nu, \alpha)$ is fitted to individual weights for the cases numbered from 1 to 80 . Each case can be identified by a combination of its scientific name and the region name of the experiment. The class and family names are also listed in the table as a reference. Species identified by scientific name are grouped into a family and several families are further grouped into a class. We can see what kind of animals are by-catch from the seabed. $N$ is the number of nonzero observations out of 27 observations in each case. The maximum likelihood estimates of the 
parameters $\hat{\nu}$ and $\hat{\alpha}$ are also listed. The last two columns give the value of goodness of fit test statistic, $\tilde{W}_{N}^{2}(\hat{\boldsymbol{\theta}})$, and the corresponding $p$-value. $p$-values less than 0.1 are marked by an asterisk in the table as a reference. It seems reasonable to exclude these cases which were not a good fit. 23 cases out of 80 cases were excluded for later analysis since we want to detect the effect of trawling through change of the equilibrium distribution of the stochastic growth model (3.2). To assist in the visual understanding of the goodness of fit of the 57 unmarked cases, several examples of P-P plots are given in Fig. 2 for Cases 1, 2 and 3.
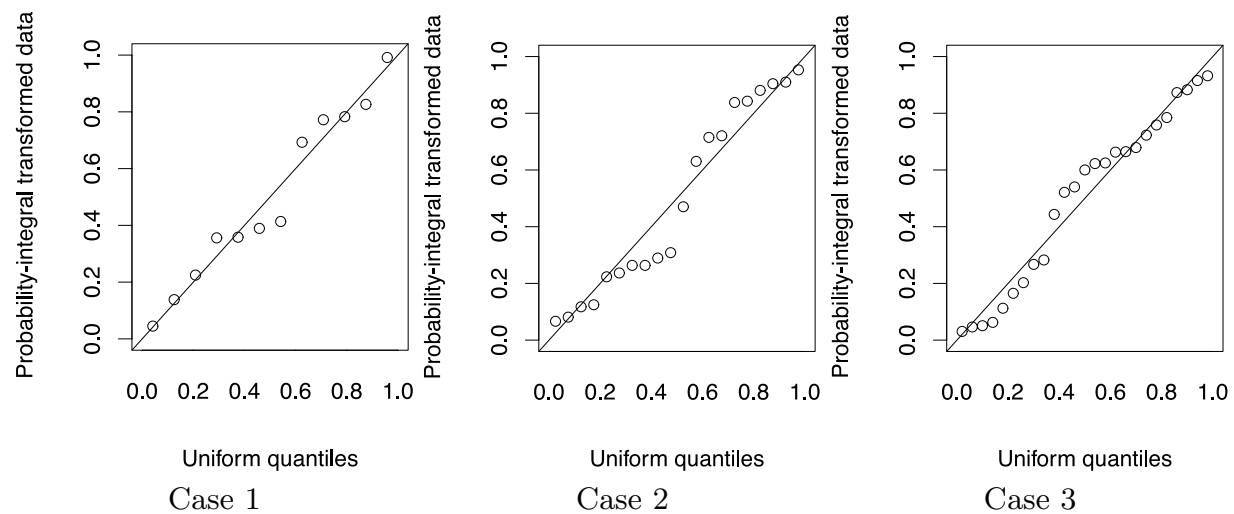

Figure 2. Examples of P-P plots for Case 1, Case 2 and Case 3.

To understand the meaning of the estimated parameters for the 57 good fit cases, a reasonable transform of the parameters would be $k=\alpha(\nu+1)$ and $\xi=\sqrt{2 /(\nu+1)}$, because it is equivalent to rewrite the model (3.2) as

$$
d X_{s}=X_{s}\left(1-\frac{X_{s}}{k}\right) d s+\xi X_{s} d B_{s}
$$

where time is changed from $t$ to $s=r t$. The parameter $k$ is now the (deterministic) limit of growth and $\xi$ is the size of random fluctuations around the limit $k$. Figure 3 is a scatter plot of $\hat{\xi}=\sqrt{2 /(\hat{\nu}+1)}$ and $\hat{k}=\hat{\alpha}(\hat{\nu}+1)$ where Case 12 is excluded because $\hat{k}=735.870$ is very large with $\hat{\xi}=1.219$. The points on the scatter plot are identified by initial letters of class names. For example, $\mathrm{H}$ is the class Hydrozoa as is described on the legend. It is easily seen that the value of $k$ is very large for 4 species, but it only implies that these species have a heavy dry weight. However, it is interesting to note that several classes share similar $\xi$ values. It is less than 0.8 for Bivalvia, greater than 1.0 for Hydrozoa and between 0.7 and 1.1 for Gymnolaemata.

The stochastic growth model (3.2) may not be a good model for some of the remaining 23 cases. In fact, only one species Dardanus imbricatus shows a good fit both in the West and East out of four species in the list for which enough observations are available both in the West and East (Cases 6, 7, 41, 42, 50, 51, 57 


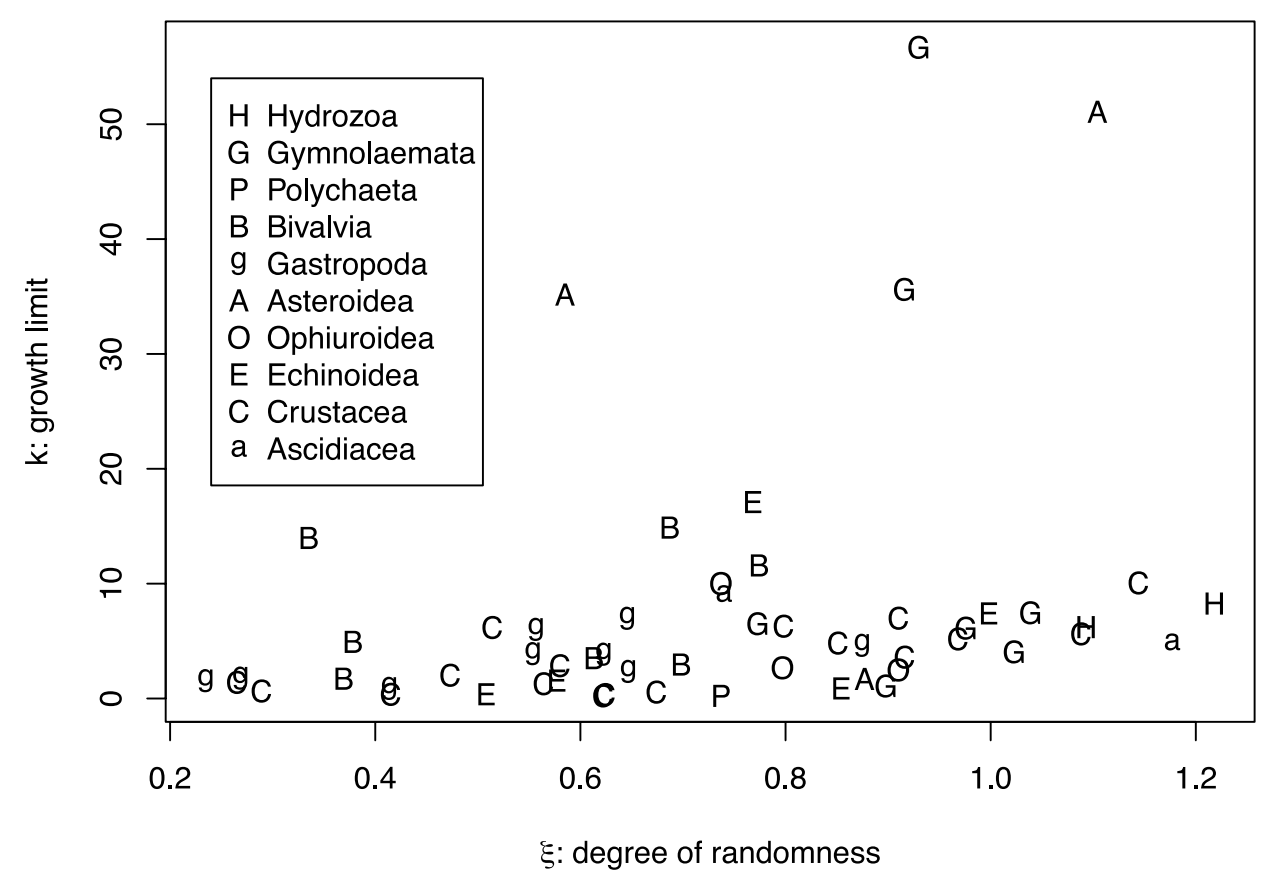

Figure 3. Scatter plot of the size of randomness $\hat{\xi}=\sqrt{2 /(\hat{\nu}+1)}$ and the growth limit $\hat{k}=\hat{\alpha}(\hat{\nu}+1)$.

and 58). Possibly, in these cases, environmental factors may have delayed species maturity or the model (3.2) does not match to those 23 cases. Also, it is worth noting that there is no consistent rejection of the goodness of fit over different species. Therefore, we are forced to consider the species by species or case by case analysis. We leave any further understanding of the results, including a biological understanding, for future investigation.

\section{Effect of trawling}

In this section we detect the effect of trawling through discrepancies in the identified gamma distribution rather than looking at gamma parameter changes. Although the discrepancy does not necessarily imply the existence of the effect of trawling, we note that the data is obtained under a careful design of experiments (Haywood et al. (2005)). Since there is no other effect than trawling we could say that we have detected the effect of trawling if the discrepancy is significant.

The statistical framework for the calculation of the $p$-value is different from that described in the previous section as no parameter estimation is involved in this stage. We calculate the $p$-value for the null distribution which is already identified before trawling. In other words, conditional $p$-values are calculated. We have simply obtained the $p$-value by generating 500 sets of random numbers distributed as the identified gamma distribution. The number of target cases now reduces to 50 since not enough individuals are available after trawling for the remaining cases. A summary of the result for the 50 cases is given in Table 3 , 
Table 3. Effect of trawling (U: Uneffected, L: Lighter, C: Changed).

\begin{tabular}{crcccccccc}
\hline Case & $\mathrm{N}$ & $\tilde{W}_{N}^{2}\left(\boldsymbol{\theta}_{0}\right)$ & $p$-value & Effect & Case & $\mathrm{N}$ & $\tilde{W}_{N}^{2}\left(\boldsymbol{\theta}_{0}\right)$ & $p$-value & Effect \\
\hline 1 & 6 & 0.092 & 0.636 & $\mathrm{U}$ & 34 & 9 & 0.115 & 0.550 & $\mathrm{U}$ \\
3 & 15 & 0.106 & 0.584 & $\mathrm{U}$ & 36 & 14 & 0.091 & 0.650 & $\mathrm{U}$ \\
5 & 9 & 0.076 & 0.734 & $\mathrm{U}$ & 40 & 7 & 0.319 & 0.106 & $\mathrm{U}$ \\
7 & 13 & 0.059 & 0.838 & $\mathrm{U}$ & 41 & 6 & 0.319 & 0.112 & $\mathrm{U}$ \\
9 & 8 & 0.077 & 0.748 & $\mathrm{U}$ & 45 & 7 & 0.096 & 0.650 & $\mathrm{U}$ \\
11 & 8 & 0.144 & 0.424 & $\mathrm{U}$ & 46 & 18 & 0.264 & 0.184 & $\mathrm{U}$ \\
12 & 6 & 0.097 & 0.604 & $\mathrm{U}$ & 47 & 11 & 0.143 & 0.404 & $\mathrm{U}$ \\
13 & 17 & 1.075 & 0.000 & $\mathrm{~L}$ & 49 & 9 & 0.658 & 0.022 & $\mathrm{~L}$ \\
14 & 8 & 0.223 & 0.212 & $\mathrm{U}$ & 51 & 13 & 0.166 & 0.348 & $\mathrm{U}$ \\
15 & 12 & 0.441 & 0.058 & $\mathrm{~L}$ & 52 & 7 & 0.367 & 0.088 & $\mathrm{~L}$ \\
19 & 9 & 0.150 & 0.422 & $\mathrm{U}$ & 53 & 12 & 1.556 & 0.000 & $\mathrm{C}(\mathrm{L})$ \\
20 & 10 & 0.044 & 0.918 & $\mathrm{U}$ & 57 & 11 & 0.211 & 0.248 & $\mathrm{U}$ \\
21 & 13 & 0.549 & 0.030 & $\mathrm{C}(\mathrm{L})$ & 58 & 8 & 0.191 & 0.276 & $\mathrm{U}$ \\
22 & 12 & 0.183 & 0.334 & $\mathrm{U}$ & 59 & 14 & 0.242 & 0.210 & $\mathrm{U}$ \\
23 & 10 & 0.446 & 0.054 & $\mathrm{~L}$ & 60 & 7 & 0.097 & 0.640 & $\mathrm{U}$ \\
24 & 12 & 0.125 & 0.530 & $\mathrm{U}$ & 61 & 12 & 0.390 & 0.094 & $\mathrm{C}$ \\
25 & 9 & 0.212 & 0.272 & $\mathrm{U}$ & 63 & 10 & 0.200 & 0.250 & $\mathrm{U}$ \\
26 & 15 & 0.458 & 0.036 & $\mathrm{C}$ & 70 & 7 & 0.066 & 0.808 & $\mathrm{U}$ \\
27 & 14 & 0.184 & 0.322 & $\mathrm{U}$ & 71 & 15 & 0.188 & 0.300 & $\mathrm{U}$ \\
28 & 8 & 0.225 & 0.208 & $\mathrm{U}$ & 73 & 6 & 0.277 & 0.136 & $\mathrm{U}$ \\
30 & 9 & 0.122 & 0.516 & $\mathrm{U}$ & 75 & 10 & 0.697 & 0.008 & $\mathrm{C}(\mathrm{L})$ \\
31 & 11 & 0.197 & 0.276 & $\mathrm{U}$ & 76 & 6 & 0.327 & 0.100 & $\mathrm{U}$ \\
32 & 18 & 0.253 & 0.210 & $\mathrm{U}$ & 78 & 12 & 0.355 & 0.118 & $\mathrm{U}$ \\
33 & 8 & 1.663 & 0.000 & $\mathrm{~L}$ & & & & & \\
\hline & & & & & & & & &
\end{tabular}

where the uneffected cases are denoted by $\mathrm{U}$, lighter cases by $\mathrm{L}$ and $\mathrm{C}$ denotes cases that change, but not consistently lighter nor heavier, in the column labelled Effect. There are no heavier cases in our study. The type of effect is determined by stochastic order. It is $\mathrm{L}$ if the discrepancy is significant with level 0.1 in terms of the $p$-value and $F_{(k)}\left(x_{(k)} ; \boldsymbol{\theta}_{0}\right)<(k-0.5) / N$ for $k=1, \ldots, N$. The latter is equivalent if all points are below the 45 degree line on the P-P plot and $\mathrm{C}(\mathrm{L})$ where it is almost L, with a few exceptional points on the P-P plot. It is worth noting that almost all species are different except Cases 57 and 58, suggesting that the effect of trawling on a species weight is different for different regions East or West.

We can see more detail about changes of the distribution through P-P plots. Figure 4 gives us such plots for 6 cases of type L. A possible interpretation for type $\mathrm{L}$ species would be that those species have difficulty avoiding the trawl net and only individuals smaller than the net size remain, so that the distribution is skewed in the lighter direction.

Figure 5 gives us the plots for 5 cases of type $\mathrm{C}$ or $\mathrm{C}(\mathrm{L})$. It is easily understood that the distribution is skewed to lighter weights for Case 21, Case 53 


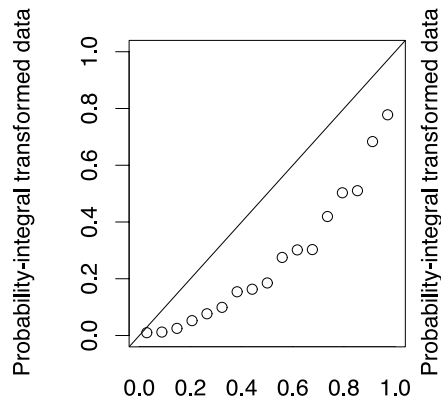

Uniform quantiles

Case 13

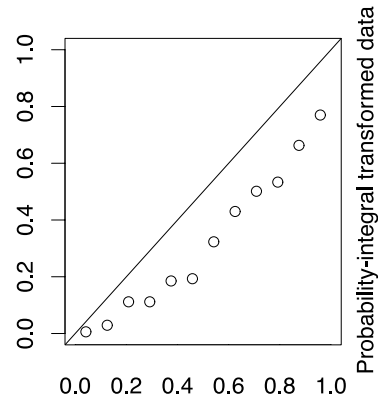

Uniform quantiles

Case 15

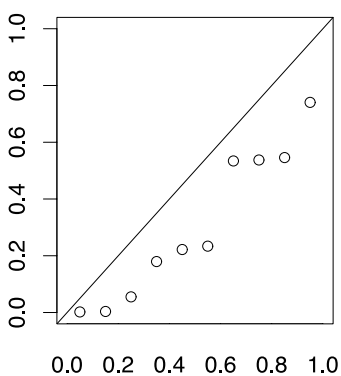

Uniform quantiles

Case 23

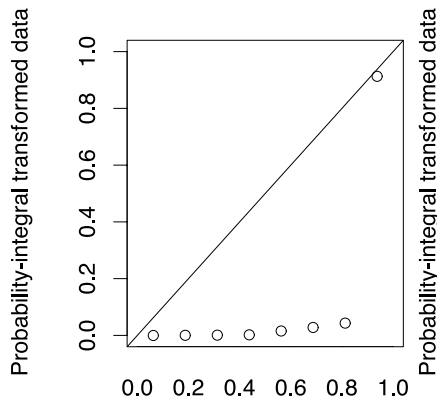

Uniform quantiles

Case 33

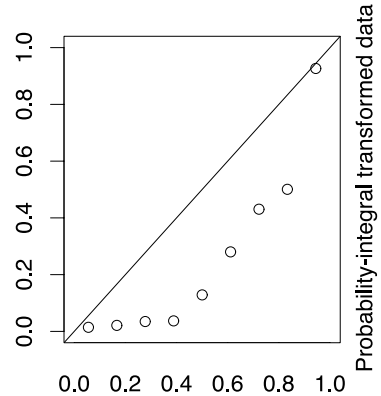

Uniform quantiles

Case 49

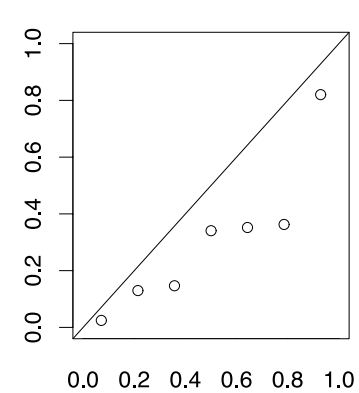

Uniform quantiles

Case 52

Figure 4. P-P plots for six cases where the weight distribution became lighter after trawling. The null distributions are $\mathrm{G}_{\mathrm{A}}(1.313,24.512), \mathrm{G}_{\mathrm{A}}(2.687,0.062), \mathrm{G}_{\mathrm{A}}(4.320,0.660)$, $\mathrm{G}_{\mathrm{A}}(35.112,0.043), \mathrm{G}_{\mathrm{A}}(2.388,5.049)$ and $\mathrm{G}_{\mathrm{A}}(5.018,0.259)$, respectively.

and Case 75, if a single point on the P-P plot was neglected. However, there is no clear direction of change for the other cases, Case 26 and Case 61. This is probably because such species are more sensitive to other factors like the local unevenness of the environment rather than that of trawling.

\section{Comparison with simple mean tests}

We have seen the effectiveness of the gamma distribution in detecting the effect of trawling. It is however worthwhile to compare this result with that obtained by a simple mean difference test statistic like Welch's $T$ as

$$
T=\frac{\bar{W}_{a}-\bar{W}_{b}}{\sqrt{\frac{s_{a}^{2}}{N_{a}}+\frac{s_{b}{ }^{2}}{N_{b}}}},
$$

where $\bar{W}_{a}$ is the sample mean of weights normalised by the number of individuals caught after trawling, $s_{a}{ }^{2}$ is the sample variance and $N_{a}$ is the sample size, and $\bar{W}_{b}, s_{b}{ }^{2}$ and $N_{b}$ are those for the normalised weights before trawling. Table 4 


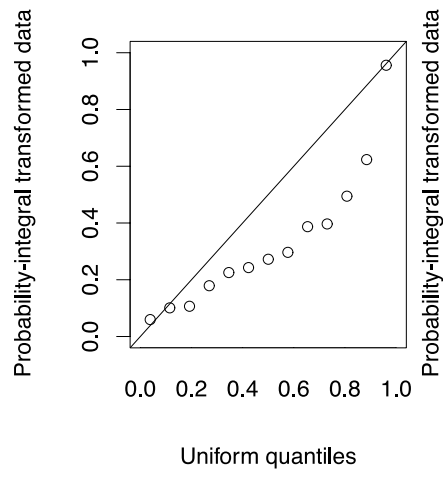

Case 21

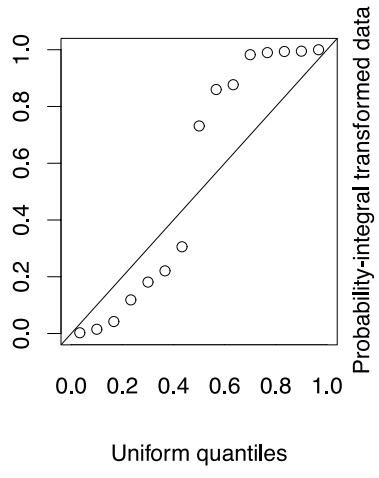

Case 26

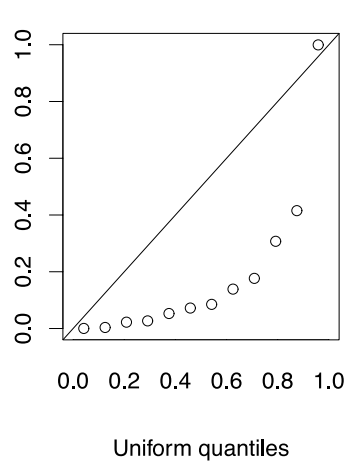

Case 53

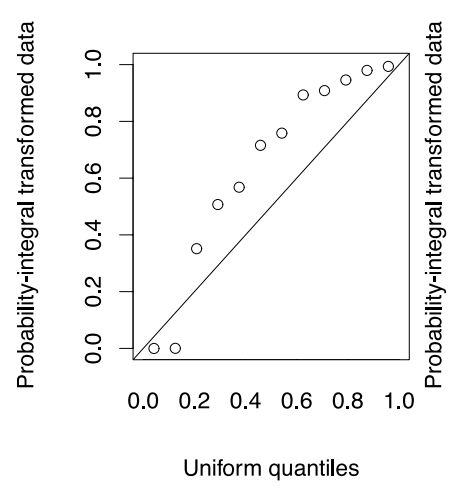

Case 61

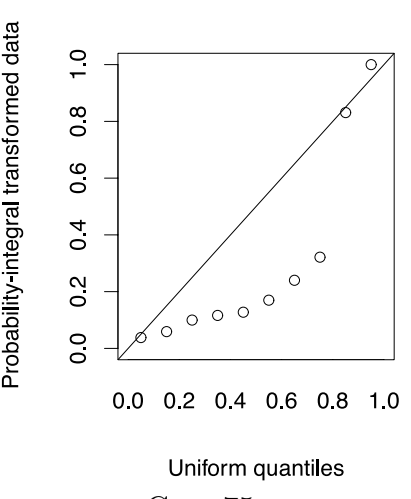

Case 75

Figure 5. P-P plots for five cases where the weight distribution changed without direction after trawling. The null distributions are $\mathrm{G}_{\mathrm{A}}(2.342,3.467), \mathrm{G}_{\mathrm{A}}(26.664,0.0463), \mathrm{G}_{\mathrm{A}}(1.007,3.693)$, $\mathrm{G}_{\mathrm{A}}(27.462,0.049)$ and $\mathrm{G}_{\mathrm{A}}(0.690,3.327)$, respectively.

shows the $p$-values for our statistic and for the $T$ statistic for the two sided alternative hypothesis in the case of type U. Also $p$-values for the Student's $T$ test statistic,

$$
T=\frac{\bar{W}_{a}-\bar{W}_{b}}{\sqrt{\frac{s_{a}^{2}}{N_{a}}}}
$$

given $\bar{W}_{b}$, are shown in the table for reference, since the $p$-values for $\tilde{W}_{N}^{2}\left(\boldsymbol{\theta}_{\mathbf{0}}\right)$ are calculated conditionally on the data before trawling.

It seems reasonable that the $p$-values for Welch's $T$ are all large for the cases of type U. However, the values themselves are not consistent with those for $\tilde{W}_{N}^{2}\left(\boldsymbol{\theta}_{\mathbf{0}}\right)$, particularly for the three cases marked $\dagger$ in the table. The reason is that the discrepancy from the null distribution is symmetric, so that the mean difference fails in describing such a discrepancy as is seen from the P-P plots given in Fig. 6. The $p$-values for the Student's $T$ are not consistent with those for Welch's $T$, particularly for the three cases marked $*$ in this table. The reason is that the conditional test is sensitive to a slight shift of the distribution as is 
Table 4. Comparison of the $p$-values for type $\mathrm{U}$.

\begin{tabular}{|c|c|c|c|c|c|c|c|c|c|}
\hline \multicolumn{3}{|c|}{ Case } & 1 & 3 & 5 & 7 & 9 & 11 & 12 \\
\hline \multicolumn{3}{|c|}{$p$-value $\left(\tilde{W}_{N}^{2}\left(\boldsymbol{\theta}_{\mathbf{0}}\right)\right)$} & 0.636 & 0.584 & 0.734 & 0.838 & 0.748 & 0.424 & 0.604 \\
\hline \multicolumn{3}{|c|}{$p$-value (Welch's $T$ ) } & 0.524 & 0.541 & 0.735 & 0.642 & 0.376 & 0.756 & 0.804 \\
\hline \multicolumn{3}{|c|}{$p$-value (Student's $T$ ) } & 0.315 & 0.428 & 0.690 & 0.507 & 0.354 & 0.734 & 0.679 \\
\hline \multicolumn{3}{|c|}{$\operatorname{sign}(T)$} & - & - & + & - & + & + & - \\
\hline 14 & 19 & 20 & 22 & 24 & 25 & 27 & 28 & 30 & $31^{*}$ \\
\hline 0.212 & 0.422 & 0.918 & 0.334 & 0.530 & 0.272 & 0.322 & 0.208 & 0.516 & 0.276 \\
\hline 0.318 & 0.777 & 0.975 & 0.757 & 0.810 & 0.465 & 0.423 & 0.628 & 0.674 & 0.154 \\
\hline 0.130 & 0.756 & 0.960 & 0.592 & 0.771 & 0.135 & 0.298 & 0.269 & 0.526 & 0.097 \\
\hline- & - & - & + & - & + & - & + & + & + \\
\hline 32 & 34 & 36 & 40 & 41 & 45 & $46^{\dagger}$ & 47 & 51 & $57^{*}$ \\
\hline 0.210 & 0.550 & 0.650 & 0.106 & 0.112 & 0.650 & 0.184 & 0.404 & 0.348 & 0.248 \\
\hline 0.851 & 0.967 & 0.721 & 0.177 & 0.260 & 0.981 & 0.992 & 0.954 & 0.393 & 0.157 \\
\hline 0.808 & 0.955 & 0.609 & 0.105 & 0.186 & 0.976 & 0.990 & 0.947 & 0.279 & 0.016 \\
\hline+ & + & - & - & + & + & - & - & - & - \\
\hline 58 & $59^{*}$ & 60 & 63 & 70 & 71 & 73 & $76^{\dagger}$ & $78^{\dagger}$ & \\
\hline 0.276 & 0.210 & 0.640 & 0.250 & 0.808 & 0.300 & 0.136 & 0.100 & 0.118 & \\
\hline 0.372 & 0.269 & 0.963 & 0.766 & 0.730 & 0.425 & 0.338 & 0.606 & 0.833 & \\
\hline 0.330 & 0.055 & 0.945 & 0.682 & 0.670 & 0.354 & 0.301 & 0.595 & 0.797 & \\
\hline- & - & + & + & - & + & - & + & - & \\
\hline
\end{tabular}

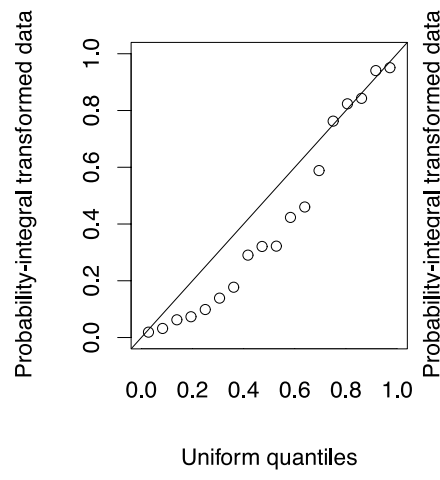

Case 46

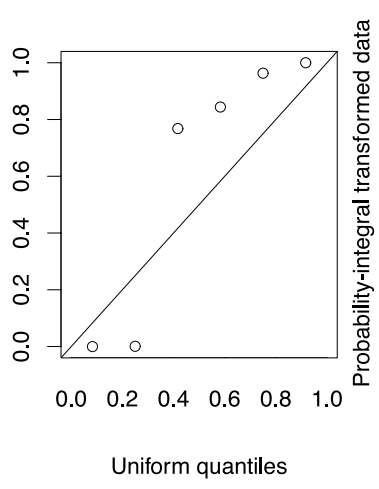

Case 76

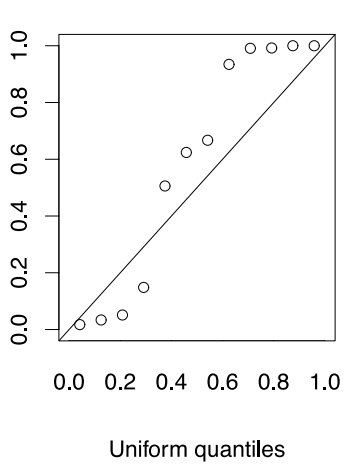

Case 78

Figure 6. P-P plots for Case 46, 76 and 78 after trawling.

seen from P-P plots for those three cases given in Fig. 7.

Table 5 is a similar table for type L. It is clear that Welch's $T$ fails in detecting changes in the 3 cases marked by $\dagger$, although the values of Welch's $T$ are all negative. The sensitivity of Student's $T$ makes a difference for Case 23 as well as for Type U.

Table 6 is for Type $\mathrm{C}$ or $\mathrm{C}(\mathrm{L})$. In this case, Welch's $T$ fails in describing 


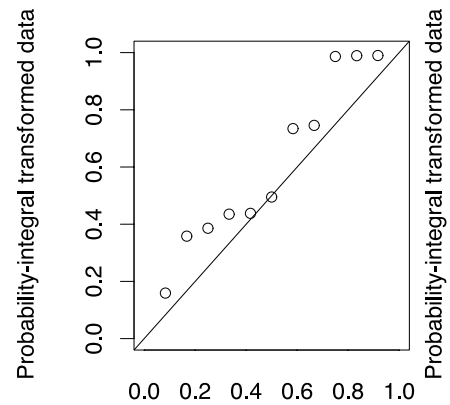

Uniform quantiles

Case 31

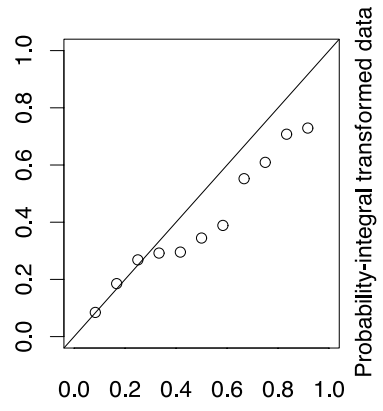

Uniform quantiles

Case 57

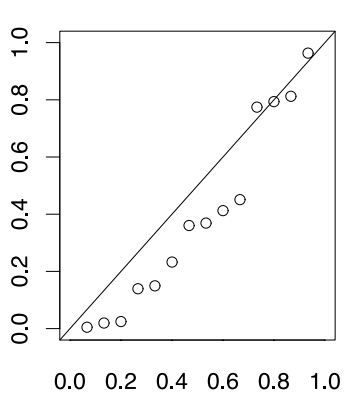

Uniform quantiles

Case 59

Figure 7. P-P plots for Case 31, 57 and 59 after trawling.

Table 5. Comparison of the $p$-values for Type L.

\begin{tabular}{lcccccc}
\hline \multicolumn{1}{c}{ Case } & 13 & $15^{\dagger}$ & $23^{\dagger}$ & 33 & 49 & $52^{\dagger}$ \\
\hline$p$-value $\left(\tilde{W}_{N}^{2}\left(\boldsymbol{\theta}_{\mathbf{0}}\right)\right)$ & 0.000 & 0.058 & 0.054 & 0.000 & 0.022 & 0.088 \\
$p$-value (Welch's $T)$ & 0.005 & 0.291 & 0.194 & 0.010 & 0.020 & 0.486 \\
$p$-value (Student's $T)$ & 0.000 & 0.174 & 0.014 & 0.009 & 0.013 & 0.384 \\
$\operatorname{sign}(T)$ & - & - & - & - & - & - \\
\hline
\end{tabular}

Table 6. Comparison of the $p$-values for Type $\mathrm{C}$ or $\mathrm{C}(\mathrm{L})$.

\begin{tabular}{lccccc}
\hline \multicolumn{1}{c}{ Case } & 21 & $26^{\dagger}$ & 53 & $61^{\dagger}$ & $75^{\dagger}$ \\
\hline$p$-value $\left(\tilde{W}_{N}^{2}\left(\boldsymbol{\theta}_{\mathbf{0}}\right)\right)$ & 0.030 & 0.036 & 0.000 & 0.094 & 0.008 \\
$p$-value (Welch's $T)$ & 0.084 & 0.168 & 0.056 & 0.937 & 0.720 \\
$p$-value (Student's $T)$ & 0.020 & 0.073 & 0.008 & 0.928 & 0.696 \\
sign $(T)$ & - & + & - & - & + \\
\hline
\end{tabular}

changes with a level of 0.1 in 3 out of the 5 cases. A significant difference is shown for Case 75, where the sign of Welch's $T$ is positive although it belongs to type $\mathrm{C}(\mathrm{L})$. Similar comments apply for the Student's $T$ as these for Type L. As a summary, Welch's $T$ tends to fail in the detection of distributional changes when the empirical distribution differs from the null distribution in a symmetric manner. On the other hand, Student's $T$ seems very sensitive for slight differences from the null distribution. Such mean difference tests are simple and easy to use, but not strong enough for detection of distributional changes since the distributions are only identified by the mean.

\section{Discussion}

We have shown that the gamma distribution, the equilibrium distribution of the stochastic growth model, not only describes the distribution of species weights but also provides good support to detect the effect of trawling for each species, 
including the direction of changes. Goodness of fit of the distribution is tested by a modified Cramér Von Mises type statistic with a graphical representation of the fit by a P-P plot. One of the reasons why we need such a test is that only the total weights of catches for each species are recorded in this survey. As a result the integrated use of numerical and graphical tests of goodness of fit provide us with a better understanding of the trawling effect. It is obvious that formal testing of gamma parameter changes does not lead us to a complete understanding of distributional changes. It is always a problem in biological data analysis to be able to cope with small numbers of effective samples. As has been shown in this paper, the introduction of a descriptive model like (3.2) can boost our analysis but it is not enough. Augmentation of data, for example, combining different treatment levels as we have done, is also needed to cope with a limited number of observations. It is of course at the cost of losing some important aspects of the data. In fact, the augmentation destroyed part of the design of experiments planned before the survey. A suggestion would then be that simple but more frequent experimental dredges are desirable for detecting the effect of trawling in future surveys.

A limitation of our analysis is that nothing can be said about the 23 species to which the equilibrium distribution does not fit well. It is a further challenge to develop another model which is applicable to these remaining species. We could classify the remaining 50 species into three types, unaffected, lighter and changed in Section 5. The fact that no heavier species were found is an interesting result which supports our analysis. However, it is unfortunate that nothing more can be said about details of the effect, for example, region specific environmental effects or ecological factors. We hope that our results will be helpful for further understanding of the effect as well as making a new sample survey.

A further challenge is to have a deeper biological understanding of the results. For example, we have discussed with biologists many times how to interpret the pair of the values of $\xi$ and $k$, which are displayed on the plot in Fig. 3. However, it was not easy for them because most of the by-catch species are new to them. We hope that our results including Table 7 will help deepen the understanding of seabed fauna in the Northern Prawn Fishery, not only from the view point of fishery, but also the view point of ecology.

\section{Appendix}

Equilibrium Distribution of the Growth Model

In this appendix, the equilibrium distribution is derived in a general frame work when $\left\{X_{t}\right\}$ satisfies

$$
d X_{t}=a\left(X_{t}\right) d t+b\left(X_{t}\right) d B_{t}
$$

It is well known that the probability density function of $p(t, x)$ for $X_{t}$ satisfies the Kolmogorov's forward equation,

$$
\frac{\partial p(t, x)}{\partial t}=-\frac{\partial}{\partial x}(a(x) p(t, x))+\frac{1}{2} \frac{\partial^{2}}{\partial x^{2}}\left(b^{2}(x) p(t, x)\right) .
$$


The equilibrium distribution $p(x)=\lim _{t \rightarrow \infty} p(t, x)$, then satisfies the equation

$$
0=-\frac{d}{d x}(a(x) p(x))+\frac{1}{2} \frac{d^{2}}{d x^{2}}\left(b^{2}(x) p(x)\right) .
$$

By integrating the both sides of (A.1), we have

$$
a(x) p(x)-\frac{1}{2} \frac{d}{d x}\left(p(x) b^{2}(x)\right)+C=0,
$$

for a constant $C$, which is not necessarily null. However, $C$ becomes 0 as far as $p(x)$ satisfies the following boundary conditions

$$
\lim _{x \rightarrow \infty} p(x) a(x)=0 \quad \text { and } \quad \lim _{x \rightarrow \infty} \frac{d}{d x}\left(p(x) b^{2}(x)\right)=0 .
$$

Then $q(x)=p(x) b^{2}(x)$ is the solution of

$$
\frac{d}{d x} q(x)-\frac{2 a(x)}{b^{2}(x)} q(x)=0,
$$

that is

$$
p(x)=\frac{C^{\prime}}{b^{2}(x)} \exp \left(\int \frac{2 a(u)}{b^{2}(u)} d u\right)
$$

where $C^{\prime}$ is a constant.

In case of our growth model, since $a(x)=r x(1-x / k)$ and $b(x)=\sigma x$, we have the gamma distribution

$$
\begin{aligned}
p(x) & =\frac{C^{\prime}}{(\sigma x)^{2}} \exp \left(\int \frac{2 r x(1-x / k)}{(\sigma x)^{2}} d x\right) \\
& =\frac{C}{\sigma^{2}} x^{-2} \exp \left(\frac{2 r}{\sigma^{2}} \int\left(\frac{1}{x}-\frac{1}{k}\right) d x\right) \\
& =\frac{1}{\alpha^{\nu} \Gamma(\nu)} x^{\nu-1} \exp \left(-\frac{x}{\alpha}\right)
\end{aligned}
$$

for $\nu=\frac{2 r}{\sigma^{2}}-1$ and $\alpha=\frac{k}{\nu+1}$.

\section{Acknowledgements}

The authors would like to thank Mr. Mick Haywood and Dr. Charis Burridge of CSIRO Marine Research for data and some helpful suggestions and the Japan Society for the Promotion of Science International Training Program for supporting M.N.'s stay in CSIRO, Australia to develop our research. 


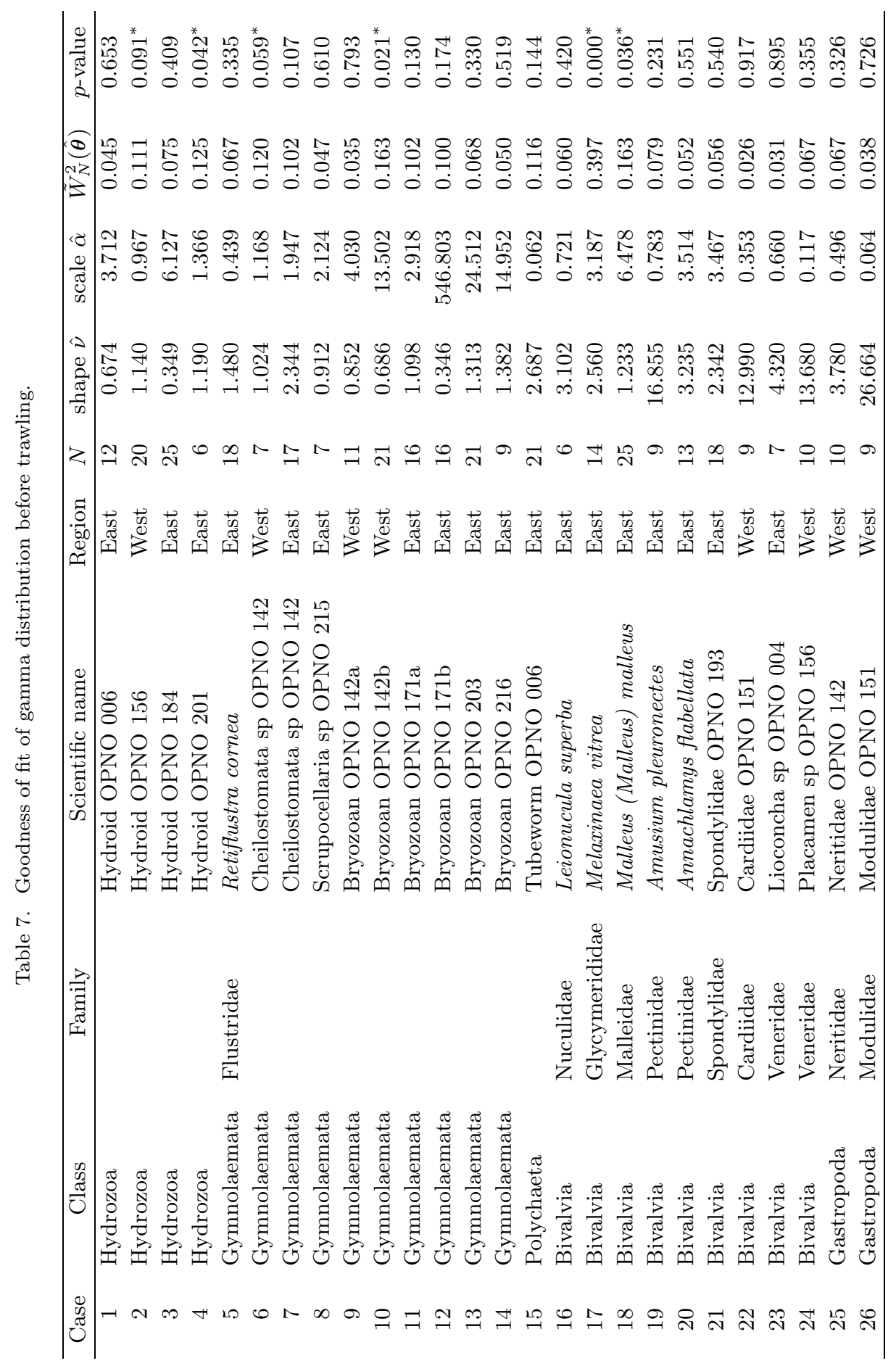




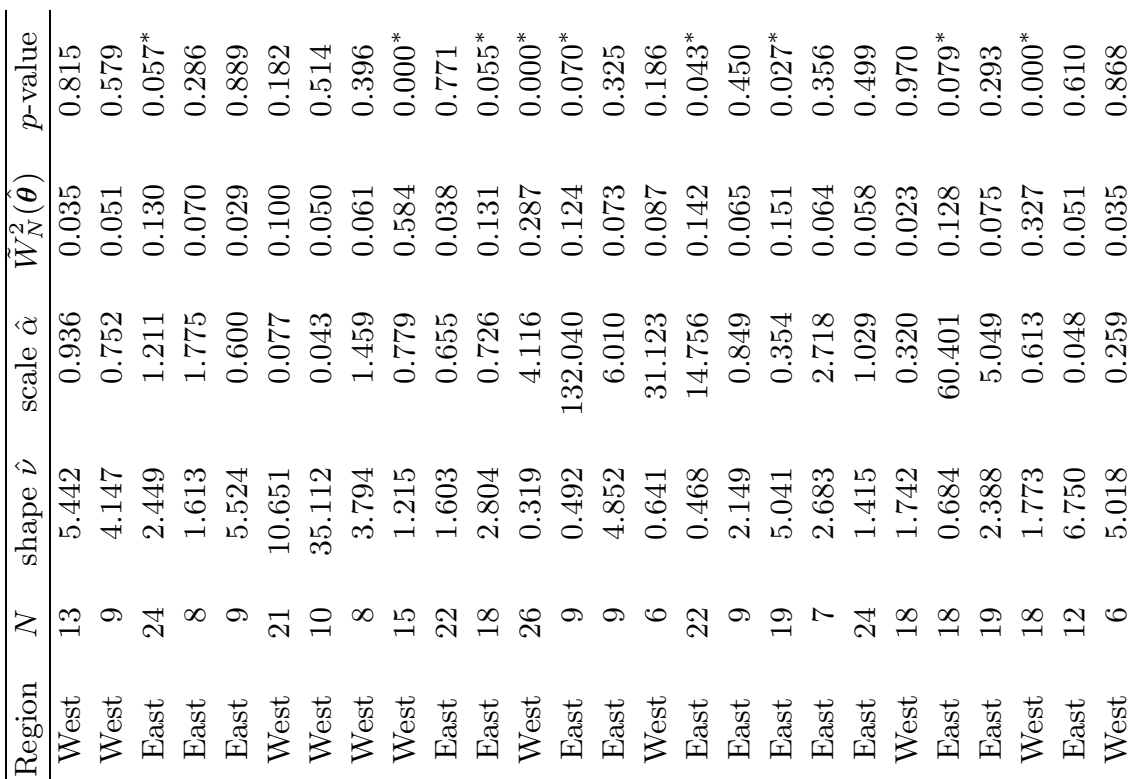

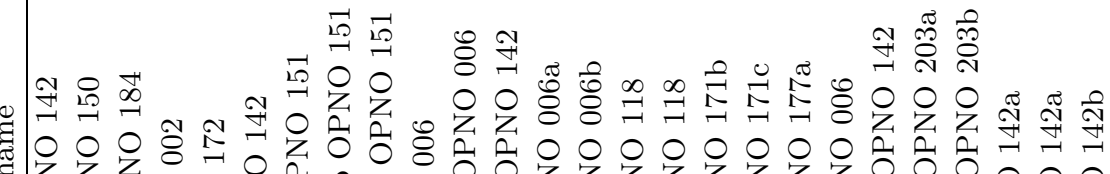
疍全

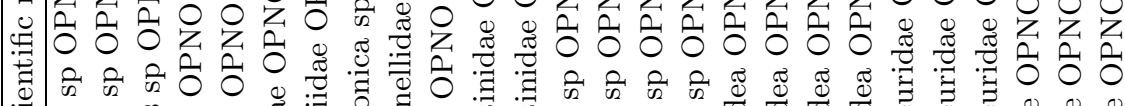

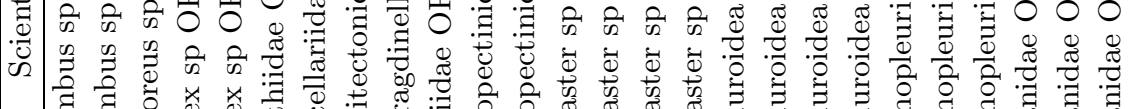

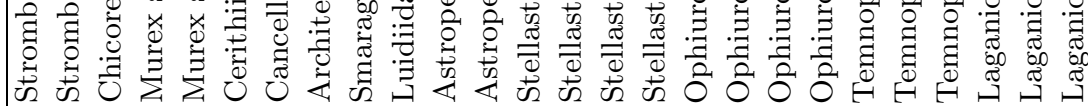

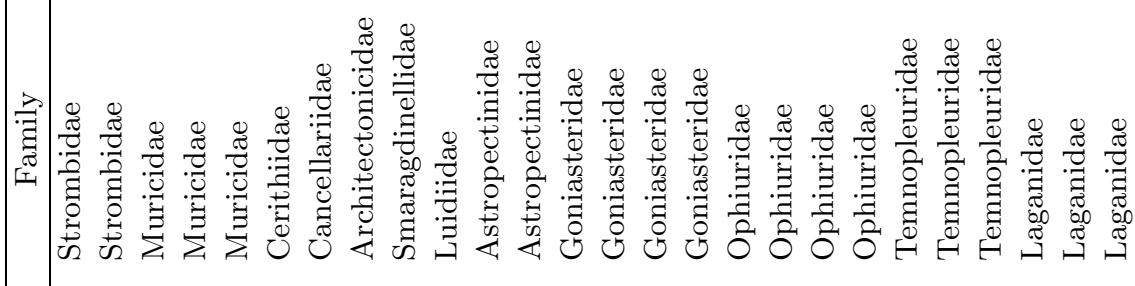

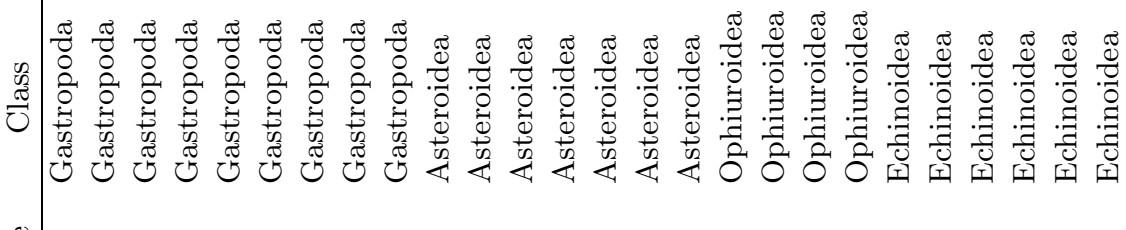

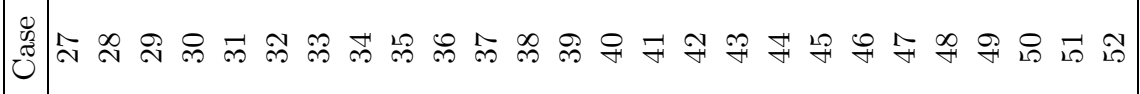




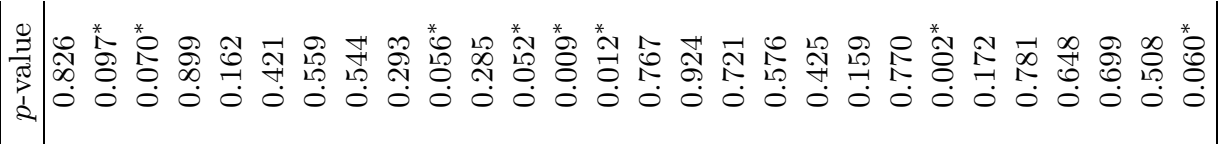

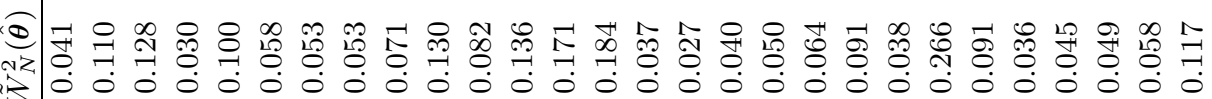

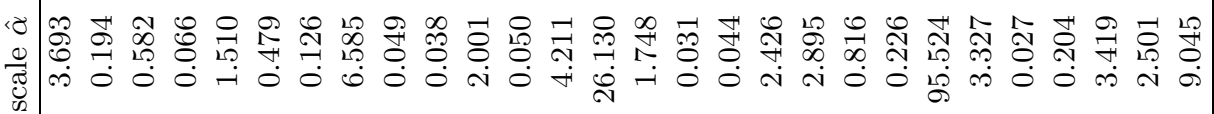

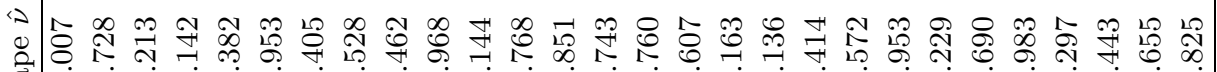
产

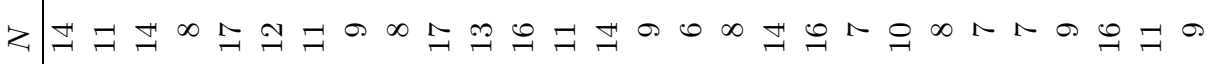

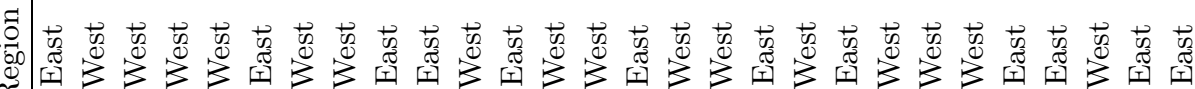

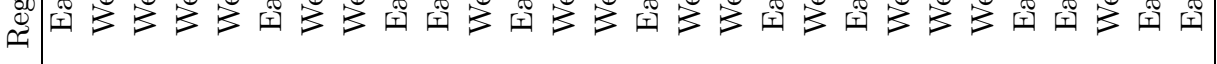

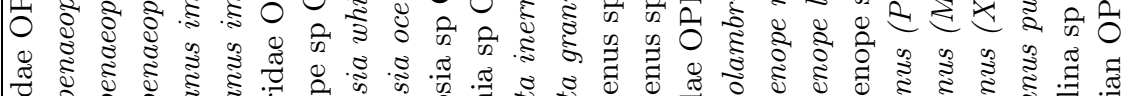

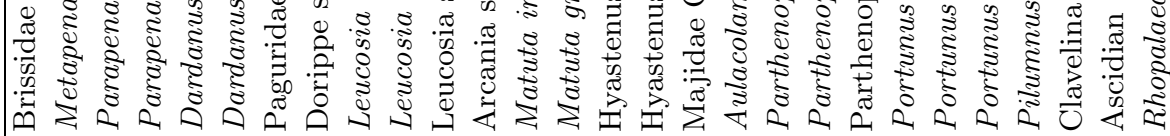

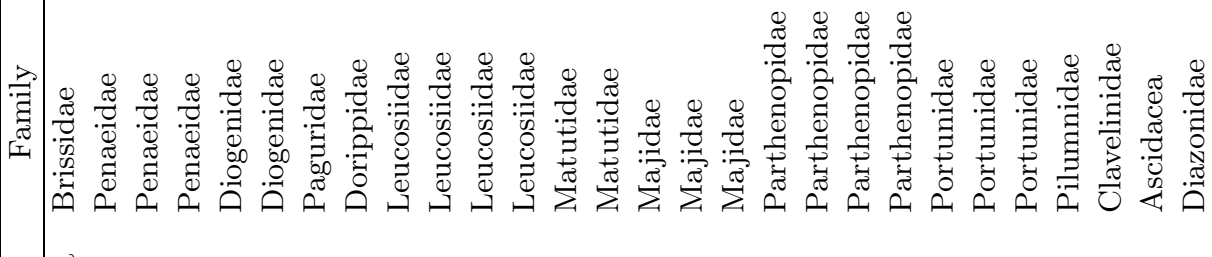

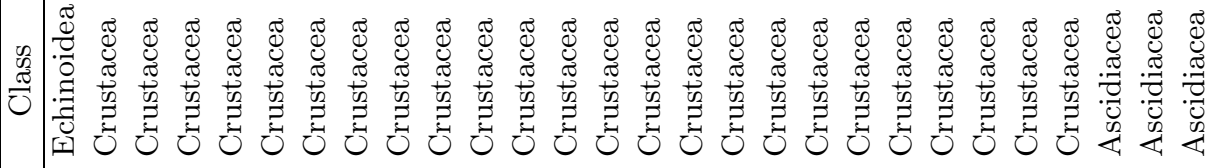

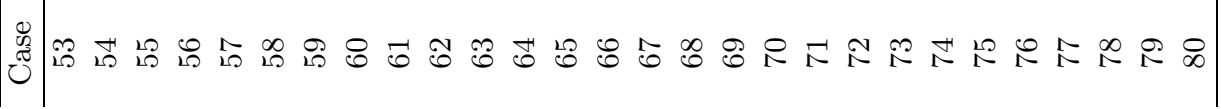




\section{REFERENCES}

Bishop, J., Die, D. and Wang, Y. (2000). Applications: A generalized estimating equations approach for analysis of the impact of new technology on a trawl fishery, Aust. N. Z. J. Stat., 42(2), 159-177.

Burridge, C., Pitcher, C., Wassenberg, T., Poiner, I. and Hill, B. (2003). Measurement of the rate of depletion of benthic fauna by prawn (shrimp) otter trawls: An experiment in the great barrier reef, Australia, Fish. Res., 60(2-3), 237-253.

Collie, J., Hall, S., Kaiser, M. and Poiner, I. (2000). A quantitative analysis of fishing impacts on shelf-sea benthos, J. Anim. Ecol., 69(5), 785-798.

Davidson, J. (1938). On the ecology of the growth of the sheep population in South Australia, T. Roy. Soc. South. Aust., 62, 141-148.

Feldman, M. W. and Roughgarden, J. (1975). A population's stationary distribution and chance of extinction in a stochastic environment with remarks on the theory of species packing, Theor. Popul. Biol., 7, 197-207.

Gan, F. F. and Koehler, K. J. (1990). Goodness-of-fit tests based on p-p probability plots, Technometrics, 32, 289-303.

Goel, N. S. and Richter-Dyn, N. (1974). Stochastic Models in Biology, Academic Press.

Haywood, M., Hill, B., Donovan, A., Rochester, W., Ellis, N., Welna, A., Gordon, S., Cheers, S., Forcey, K., McLeod, I., Moeseneder, C., Smith, G., Manson, F., Wassenberg, T., Thomas, S., Kuhnert, P., Laslett, G., Burridge, C. and Thomas, S. (2005). Quantifying the Effects of Trawling on Seabed Fauna in Northern Prawn Fishery, FRDC Project No. 2002/102, CSIRO Marine Research, Cleveland.

Holmgren, E. B. (1995). The p-p plot as a method for comparing treatment effects, J. Am. Stat. Assoc., 90(429), 360-365.

Lockhart, R. A. and Stephens, M. A. (1985). Goodness-of-fit tests for the gamma distribution, Technical report, Simon Fraser University.

Loynes, R. M. (1980). The empirical distribution function of residuals from generalised regression, Ann. Stat., 8(2), 285-298.

Luceño, A. (2007). A universal qq-plot for continuous non-homogeneous populations, J. Appl. Stat., 34(10), 1207-1223.

Marubini, E., Resele, L. F., Tanner, J. M. and Whitehouse, R. H. (1972). The fit of Gompertz and logistic curves to longitudinal data during adolescence on height, sitting height and biacromial diameter in boys and girls of the Harpenden Growth Study, Hum. Biol., 44(3), $511-523$.

May, R. M. (1973). Stability in randomly fluctuating versus deterministic environments, Am. Nat., 107(957), 621-650.

Pierce, D. A. and Kopecky, K. J. (1979). Testing goodness of fit for the distribution of errors in regression models, Biometrika, 66(1), 1-5.

Richards, F. J. (1969). The quantitative analysis of plant growth, Plant Physiology, Academic Press, 3-76.

Rupsys, P. and Petrauskas, J. (2007). The relationships between the diameter growth and distribution laws, WSEAS Trans. Biol. Biomed., 4, 142-161.

Russo, T., Baldi, P., Parisi, A., Magnifico, G., Mariani, S., and Cataudella, S. (2009). Lévy processes and stochastic von Bertalanffy models of growth, with application to fish population analysis, J. Theor. Biol., 258(4), 521-529.

Smith, F. E. (1963). Population dynamics in Daphnia magna and a new model for population growth, Ecology, 44(4), 651-663.

Sukhatme, S. (1972). Fredholm determinant of a positive definite kernel of a special type and its application, Ann. Math. Stat., 43(6), 1914-1926.

Tovar-Ávila, J., Troynikov, V., Walker, T. and Day, R. (2009). Use of stochastic models to estimate the growth of the Port Jackson shark, Heterodontus portusjacksoni, off eastern Victoria, Australia, Fish. Res., 95(2-3), 230-235. 
Verhulst, P. F. (1838). Notice sur la loi que la population suit dans son accroissement, Corresp. Math. Phys., 10, 113-121.

von Bertalanffy, L. (1960). Fundamental aspects of normal and malignant growth, Principles and Theory of Growth, New York, Elsevier, 137-259. 